\title{
Roteiro para Aplicação Do Lean Seis Sigma na Melhoria de Processos Industriais
}

\author{
RESUMO
}

Ariane Ferreira Porto Rosa afprosa61@gmail.com

Universidade Federal de Pelotas (UFPel), Pelotas, Rio Grande do Sul, Brasil

Rafael dos Santos Souza rsouza.eim@gmail.com

Universidade Federal de Pelotas (UFPel), Pelotas, Rio Grande do Sul, Brasil

Rogério Royer

rogroyer@ufrgs.b

Universidade Federal de Pelotas (UFPel),

Pelotas, Rio Grande do Sul, Brasil
Os conceitos acerca das práticas enxutas do Lean Manufacturing assim como as ferramentas estatísticas do Seis Sigma possuem ampla difusão por parte da comunidade acadêmica. O presente artigo, visa discutir sobre a união dos conceitos estatísticos fortemente explorados no Seis Sigma aliados aos princípios de eliminação de desperdícios do Lean Manufacturing com a finalidade de promover a melhoria do processo produtivo por meio da abordagem Lean Seis Sigma. Como objetivo, este artigo busca, a partir de uma breve revisão teórica, apresentar os conceitos, métodos, procedimentos das etapas e ferramentas da qualidade propostas no Seis Sigma, das ferramentas propostas no Lean Manufacturing, assim como a integração de ambas no Lean Seis Sigma. Serão apresentados também alguns exemplos da aplicação do Lean Seis Sigma em estudos de caso da literatura. Após isso, será proposto um roteiro definindo os passos para aplicação da integração das ferramentas Lean Seis Sigma aplicadas na obtenção de melhorias em processos industriais.

PALAVRAS-CHAVE: Seis sigma. Lean Manufacturing. Lean seis sigma. Processos industriais. 


\section{INTRODUÇÃO}

Segundo Spina (2007), a metodologia Seis Sigma (SS) está se consolidando no mundo dos negócios e proporcionando excelentes resultados às empresas. O SS é utilizado para reduzir a variabilidade dos processos, e a finalidade de atingir níveis de defeitos próximos a zero na otimização do processo.

Atualmente a aplicação do Lean Manufacturing (LM) em empresas vem crescendo, como também expandindo para outros setores além de manufatura, como na prestação de serviços (GIANNINI, 2007). O LM visa na eliminação dos desperdícios, o que não agrega valor para cliente e aumenta a velocidade dos processos produtivos.

A conceituação estatística fortemente explorada no SS aliada aos princípios do LM tem sido usada com a finalidade de promover a melhoria do processo produtivo por meio da abordagem integrada Lean Seis Sigma (LSS).

Segundo Werkema (2008), o LSS pode e deve ser utilizado por empresas de qualquer setor. Para Rotondaro (2002), podemos entender o LSS como uma metodologia com estrutura de trabalho definida e com foco na melhoria contínua de processos.

Assim, o LSS possibilita o uso de ferramentas visando melhorar o desempenho do negócio, tendo seus impactos focados na redução da variação dos processos, redução do tempo de lançamento de novos produtos, redução de custos, aumento do nível de satisfação dos clientes, redução de estoques, eliminação de desperdícios, ou seja, na otimização da organização como um todo.

Spina (2007) apresentou um roteiro de aplicação de conceitos e ferramentas de LSS em um processo de serviço, com a utilização de um software de modelagem e simulação. O objetivo de seu trabalho foi analisar as vantagens e desvantagens da utilização da simulação de processos como ferramenta na aplicação da metodologia LSS.

No presente trabalho busca-se apresentar um roteiro original para aplicação do LSS em processos de manufatura industriais e não no setor de serviços.

Para Reis (2003), verifica-se que a metodologia LSS vem sendo introduzida no Brasil por empresas multinacionais ou globais de manufatura que utilizam a metodologia em seu país de origem e, por esta razão, tentam aplicá-la à realidade brasileira. Entretanto, muito pouco, porém, é divulgado com o nível de detalhes desejado, com relação ao sucesso ou fracasso dessas iniciativas.

A proposição de um roteiro original para aplicação do LSS em processos de manufatura permitirá sistematizar os passos para aplicação de ferramentas de LSS e poderá contribuir significativamente para fontes de informação teóricas sobre o tema da melhoria de processos, bem como elevará o nível de conhecimento do assunto Lean Seis Sigma no escopo abrangido pelo trabalho.

Nesse trabalho, inicialmente será realizado uma breve revisão teórica dos conceitos, métodos, procedimentos das etapas e ferramentas da qualidade propostas no SS assim como das ferramentas propostas no LM. Serão apresentados também alguns exemplos da aplicação do LSS em estudos de caso da literatura. Após isso, será apresentada uma proposta metodológica para 
implantação do LSS através de um roteiro simples e acessível compreensão, porém original, constituído de 5 Etapas e 11 Passos.

\section{REVISÃO TEÓRICA}

Esta seção apresenta uma breve revisão teórica dos conceitos, métodos, procedimentos das etapas e ferramentas da qualidade propostas no SS assim como das ferramentas propostas no LM. Serão apresentados também alguns exemplos da aplicação do LSS em estudos de caso da literatura.

\section{LEAN MANUFACTURING}

LM também conhecido como Sistema Toyota de Produção ou Produção Enxuta foi aplicado primeiramente pela Toyota Motor Company, surgiu no Japão em meados da década de 1950 no período pós Segundo Guerra Mundial, pelo Engenheiro Taiichi Ohno (GREEF et al., 2012).

O conceito LM se disseminou internacionalmente por suas características e várias são as definições empregadas. Segundo Rago (2003), o LM apresenta exuberância de processos flexíveis, o que permite a produção ao menor custo, de modo a eliminar as perdas. Já para Ohno (1997) visa também em atender as expectativas dos clientes no menor tempo possível e com extrema qualidade. A ideia básica é produzir apenas o necessário, no momento necessário e na quantidade requerida.

Para se alcançar um estado de produção enxuta, é necessário identificar as atividades envolvidas no processo produtivo classificando-as naquelas que agregam ou não valor, de modo a eliminar os desperdícios. Segundo Shingo (1996), todas as atividades que não agregam valor, são categorizadas como perdas.

No Quadro1, são descritas as sete perdas no processo produtivo segundo Shingo (1996) e Ohno (1997).

Quadro 1 - Sete perdas do processo produtivo

\begin{tabular}{|c|c|}
\hline 7 Perdas & \multicolumn{1}{c|}{ Significado } \\
\hline Superprodução & $\begin{array}{r}\text { Relacionada à quantidade, refere-se à produção em excesso, } \\
\text { produzir em quantidades superiores a do volume programado. }\end{array}$ \\
\hline Espera & $\begin{array}{r}\text { Consiste no tempo de ociosidade seja de pessoas, informações ou } \\
\text { peças, que impossibilitam o início do próximo processo. }\end{array}$ \\
\hline Transporte & Refere-se ao excesso de movimentos seja de pessoas, peças ou \\
informações.
\end{tabular}




\begin{tabular}{|c|c|}
\hline Movimento & $\begin{array}{c}\text { Relacionada à desorganização do layout fabril, resultando em } \\
\text { movimentos desnecessários realizados pelo operador em suas } \\
\text { atividades. }\end{array}$ \\
\hline $\begin{array}{c}\text { Fabricação de } \\
\text { peças e } \\
\text { produtos } \\
\text { defeituosos }\end{array}$ & $\begin{array}{c}\text { Relacionada à fabricação de produtos que apresentam } \\
\text { características de qualidade fora do especificado, ou seja, que não } \\
\text { atendem as especificações dos clientes. }\end{array}$ \\
\hline
\end{tabular}

Fonte: Adaptado de Shingo (1996) e Ohno (1997).

George (2004) define o conceito LM como um conjunto de princípios, com o objetivo de aumentar a velocidade de todos os processos da empresa. O LM de forma sintetizada consiste na implementação de um conjunto de técnicas e ferramentas focadas na otimização de processos e na redução de desperdícios. Este conjunto de técnicas e ferramentas precisa ser cuidadosamente aplicado de modo a atender as necessidades da empresa.

No Quadro 2 são descritas algumas ferramentas usadas para colocar em prática o LM.

Quadro 2- Ferramentas do Lean Manufacturing

\begin{tabular}{|c|c|c|}
\hline Ferramentas & Conceito & Objetivo \\
\hline $\begin{array}{c}\text { Mapa Fluxo de } \\
\text { Valor }\end{array}$ & $\begin{array}{l}\text { Ferramenta que utiliza símbolos } \\
\text { e ícones para documentar e } \\
\text { ilustrar a sequencia e o } \\
\text { movimento de materiais, } \\
\text { informações e ações que } \\
\text { consiste no fluxo de valor de } \\
\text { uma empresa (WERKEMA, } \\
\text { 2011). }\end{array}$ & $\begin{array}{c}\text { Permite analisar e diagnosticar o } \\
\text { mapa do fluxo de valor do estado } \\
\text { atual da linha produtiva, de modo } \\
\text { a identificar as oportunidades de } \\
\text { melhorias. Em seguida, contribui } \\
\text { para o planejamento do mapa do } \\
\text { estado futuro de como a empresa } \\
\text { devera fluir. }\end{array}$ \\
\hline Kaizen & $\begin{array}{c}\text { Melhoramento contínuo visa o } \\
\text { alcance de melhorias rápidas } \\
\text { que compreende no emprego } \\
\text { organizado do senso comum e } \\
\text { da criatividade dos } \\
\text { colaboradores, de modo a } \\
\text { melhorar o processo ou fluxo } \\
\text { de valor completo (WERKEMA, } \\
\text { 2011). }\end{array}$ & $\begin{array}{l}\text { A melhoria contínua, toda vez que } \\
\text { se realiza uma tarefa repetida, é } \\
\text { importante realizá-la com a } \\
\text { melhor qualidade, no menor } \\
\text { tempo, comparado a última vez, } \\
\text { consiste no aprendizado com a } \\
\text { repetitividade no processo. }\end{array}$ \\
\hline Kanban & $\begin{array}{l}\text { Um dispositivo sinalizador que } \\
\text { autoriza e da instrução para a } \\
\text { produção ou retirada de itens } \\
\text { para sistema puxado (LÉXICO } \\
\text { LEAN, 2003). }\end{array}$ & $\begin{array}{l}\text { Evitar os excessos de produção, } \\
\text { de modo a eliminar desperdícios e } \\
\text { restabelecer o nível de estoque de } \\
\text { acordo com a demanda. }\end{array}$ \\
\hline Poka-Yoke & $\begin{array}{l}\text { Um dispositivo a prova de erros } \\
\text { ou falhas, é qualquer } \\
\text { mecanismo que evite que o } \\
\text { erro acorra e torna-se um } \\
\text { defeito (WERKEMA, 2011). }\end{array}$ & $\begin{array}{l}\text { Detectar ou corrigir as falhas que } \\
\text { podem ocorrer durante o } \\
\text { processo de manufatura, antes } \\
\text { que seja percebida pelos clientes. }\end{array}$ \\
\hline
\end{tabular}

Fonte: Autoria Própria 


\section{SEIS SIGMA}

O SS nasceu na Motorola durante a segunda metade dos anos oitenta, criado pelo engenheiro Bill Smith, com objetivo de alcançar altos níveis de qualidade e elevar a competitividade da empresa no mercado externo (WERKEMA, 2002).

A metodologia SS tem como princípio reduzir as quantidades de defeitos nos processos. Nos dias de hoje, o SS é apresentado como uma atividade estratégica, que retém uma abordagem de negócio em medições financeiras. O SS é uma metodologia estruturada que desenvolve a qualidade através da melhoria continua dos processos, seja na produção de um bem ou serviço (ROTONDARO, 2002).

Entretanto, para Werkema (2002), o SS está mais relacionado a uma estratégia gerencial, extremamente quantitativa, onde visa, aumentar rapidamente a lucratividade da empresa, através da qualidade de produtos e processos.

Segundo Pandeet al. (2001) a estratégia SS apresenta um importante ciclo de melhoria conhecido como DMAIC, um método baseado na resolução de problemas. De acordo com Werkema (2002), o DMAIC é dividido em cinco etapas que são descritos na Figura 1.

Figura 1- Etapas da metodologia DMAIC

SIGLAS ETAPAS OBJETIVOS

\begin{tabular}{|c|c|c|c|}
\hline D & $\rightarrow$ & Define (Definir) & Detalhar o problema e definir a meta do projeto. \\
\hline M & $\rightarrow$ & Measure (Medir) & $\begin{array}{l}\text { Mesurar o desempenho do processo, que durante a coleta de } \\
\text { dados possibilita obter as primeiras ideias da causa do problema e } \\
\text { identificar os pontos fortes e as oportunidades para sua melhoria. }\end{array}$ \\
\hline A & $\rightarrow$ & Analyse (Analisar) & $\begin{array}{l}\text { Analisar os dados coletados na fase anterior, identificar e organizar } \\
\text { as causas potenciais do problema quantificar a importância das } \\
\text { causas potenciais. }\end{array}$ \\
\hline $\mathbf{I}$ & $\rightarrow$ & Improve (Melhorar) & $\begin{array}{l}\text { Propor, avaliar e implementar soluções potenciais com o objetivo } \\
\text { de eliminar as causa fundamentais do problema prioritário analisado } \\
\text { na fase anterior. }\end{array}$ \\
\hline C & $\rightarrow$ & Control (Controlar) & $\begin{array}{l}\text { Garatir o alcance da meta em longo prazo. São adotadas diversas } \\
\text { ferramentas para controlar continuamente o desempenho dos } \\
\text { processos. }\end{array}$ \\
\hline
\end{tabular}

Fonte: WERKEMA (2002)

A metodologia SS utiliza-se de várias métricas comuns na qualidade para reduzir as quantidades de defeitos nos processo produtos e serviços. Apesar de variações existentes, segundo Pandeet al. (2001), existem algumas métricas principais definidas a seguir.

Uma primeira métrica é a medida da Proporção Defeituosa, p, calculada de acordo com a equação (1). 


$$
p=\frac{\text { Número Total de Defeituosos }}{\text { Número Total de Unidades }}
$$

A métrica prefere-se à fração ou percentual de amostras do item, que contenha um ou mais defeitos, conforme demonstrado na equação (1).

Outra métrica é o Rendimento Final, expresso na equação (2).

Rendimento Final=1-(Proporção Defeituosa)

O Rendimento Final é calculado como 1 menos a proporção defeituosa.

A métrica Defeito Por Unidades ou DPU é apresentada na equação (3).

$$
\text { DPU }=\frac{\text { Número Total de Defeitos }}{\text { Número Total de Unidades }}
$$

O DPU refere-se a o número médio de defeitos, de qualquer tipo, sobre o número total de unidades da amostra.

A proporção de Defeito por unidade, ou DPO, é apresentada na equação (4).

$$
\text { DPO }=\frac{\text { Número Total de Defeitos }}{\text { Número Total de Unidades } \times \text { Número de Oportunidades de Defeito }}
$$

O DPO refere-se à proporção de defeitos em relação ao número total de oportunidades em um grupo.

O Defeito por Milhão de Oportunidades, ou DPMO é apresentado na equação (5).

$$
\mathrm{DPMO}=\mathrm{DPO} \times 1.000 .000
$$

A maioria das medidas de oportunidades para defeitos é traduzida dessa forma em DPMO, que significa quantos defeitos surgiriam se houvesse um milhão de oportunidades.

O rendimento de primeira passada, ou RPP, é apresentado na equação (6) e refere-se à probabilidade de um produto sair com zero defeito.

$$
\mathbf{R P P}=1-\frac{\text { Número de Unidades Retrabalhadas }}{\text { Número de Unidades que Entraram }}
$$

De acordo com Einset e Marzano (2002), estima-se que partes das empresas trabalham em um nível de qualidade três sigma, o que representa um custo de $20 \%$ do faturamento em perdas, retrabalho, desperdícios e perca de clientes. Estima-se que com a simples aplicação e monitoramento das métricas propostas na metodologia Seis Sigma os custos podem ser reduzidos em um curto período.

\section{INTEGRAÇÃO LEAN SEIS SIGMA}

A Expressão "Lean Seis Sigma", ou também conhecida como "Lean Sigma", surgiu na segunda metade dos anos 90 , no momento que as empresas da cadeia automotiva (fornecedores e montadores), foram as primeiras a implementar o 
programa LM na década de 80 , passaram também a adotar os princípios da metodologia SS (FONTE, 2008).

A integração entre LM e SS é natural e não possui conflitos, pois ambas estão voltadas para a promoção da melhoria contínua na organização. A melhor forma de integrá-los é aplicando os pontos fortes de cada uma das metodologias (WERKEMA, 2011).

Segundo Pannell, (2006) as duas metodologias juntas são uma poderosa ferramenta que se completam e não competem entre si. O fato não está em usar uma metodologia ou outra, mas usar ambas as técnicas em conjunto, podendo ser essencial para uma completa estratégia de melhoria de desempenho, no período de longo prazo.

As empresas devem aproveitar os pontos fortes de cada metodologia. Por exemplo, o LM não possui um método estruturado e sistemático para solução de problemas, não consegue colocar um processo em controle estatístico, de modo diminuir a variabilidade, características que constituem a metodologia Seis Sigma. Já o SS não consegue imprimir velocidade nos processos, reduzir o lead time, características que complementam o LM (WERKEMA, 2011).

De acordo com Werkema (2011) os pontos fortes do LM e SS, são ilustrados na Figura 2.

Figura 2- Os pontos fortes do Seis Sigma e do Lean Manufacturing.

\begin{tabular}{|c|c|}
\hline SEIS SIGMA & LEAN MANUFACTURING \\
\hline $\begin{array}{l}\text { 1. Utilização, para a análise de dados, de } \\
\text { ferramentas estatística poderosa, que } \\
\text { auxiliem na solução de problemas } \\
\text { complexos }\end{array}$ & $\begin{array}{l}\text { 1. Tendência para ação imediata no caso da } \\
\text { solução de problemas de escopo restrito } \\
\text { e de baixa complexidade Kaizen. }\end{array}$ \\
\hline $\begin{array}{l}\text { 2. Busca da redução da variabilidade. } \\
\text { 3. Ênfase na redução de custos e defeitos } \\
\text { definidos pelos clientes. }\end{array}$ & $\begin{array}{l}\text { 2. Utilização de Técnicas simples para } \\
\text { análise de dados durante os eventos } \\
\text { kaizen. }\end{array}$ \\
\hline $\begin{array}{l}\text { 4. Seleção de projetos associados ás metas } \\
\text { estratégicas da empresa. }\end{array}$ & $\begin{array}{l}\text { 3. Busca da redução do lead time e do } \\
\text { trabalho em processo. }\end{array}$ \\
\hline $\begin{array}{l}\text { 5. Mensuração direta do retorno financeiro } \\
\text { gerado pelo programa. }\end{array}$ & $\begin{array}{l}\text { 4. Ênfase na maximização da velocidade dos } \\
\text { processos }\end{array}$ \\
\hline $\begin{array}{l}\text { 6. Elevada dedicação dos especialistas. } \\
\text { 7. Envolvimento de todas as pessoas da } \\
\text { empresa, diferenets níveis de } \\
\text { aprofundamento do programa (black Belts, } \\
\text { Green Belts, Yellow Belts), como } \\
\text { responsáveis por conhecer e implementar } \\
\text { seus conceitos e sua metodologia. }\end{array}$ & $\begin{array}{l}\text { 5. Seleção de projetos estratégicos } \\
\text { identificados pelo Mapeamento do Fluxo } \\
\text { de Valor e também de projetos de } \\
\text { interesse exclusivo para alguma área da } \\
\text { empresa. }\end{array}$ \\
\hline
\end{tabular}

Fonte: Werkema (2011)

A integração entre LMe SS resulta em um programa que unifica os pontos fortes de cada uma das metodologias, chamado de Lean Seis Sigma (LSS). O LSS é uma estratégia gerencial altamente quantitativa e eficaz. O seu objetivo é o aumento do desempenho e da lucratividade da empresa. Cada uma das partes da metodologia está direcionada na solução de todos os tipos de problemas relacionados a processos ou produtos (WERKEMA, 2011).

O LSS baseia-se em uma metodologia ainda mais ampla, em que é possível combinar e aplicar diversas ferramentas de ambas as metodologias, de acordo com a necessidade e o nível de qualidade empresarial. 
A Figura 3 ilustra as técnicas e ferramentas do LM, do SS e as ferramentas em comum entre cada metodologia.

Figura 3- Técnicas e ferramentas de Lean e Seis Sigma

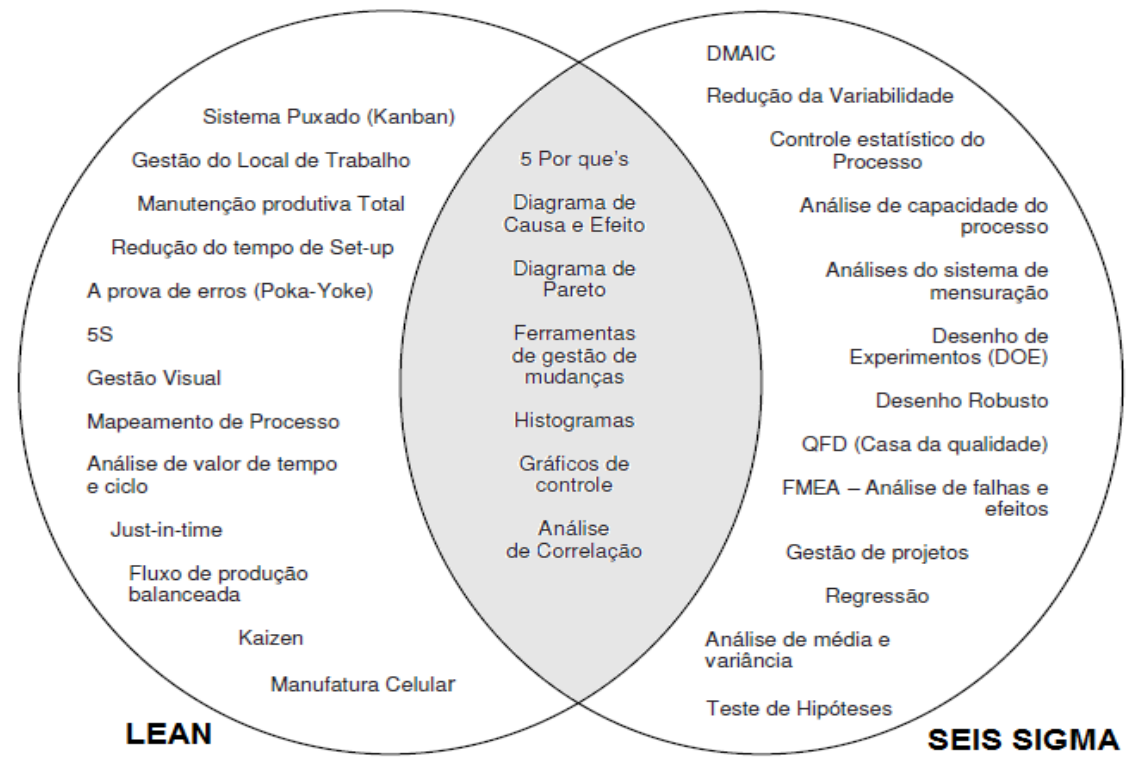

Fonte: Kumar (2006)

\section{CASOS DE APLICAÇÃO DO LEAN SEIS SIGMA}

A seguir serão descritosalguns trabalhos encontrados na literatura sobre aplicação de LSS em empresas do setor industrial.

Kuniyoshi (2006) explorou a implementação da metodologia LSS, destacando suas vantagens na obtenção de resultados. $O$ estudo de caso em uma empresa do setor têxtil, demostrou que a utilização do LSS resultou em melhorias e que pode ser aplicada em diferentes casos.

Kumar et al. (2006), analisou a aplicação de ferramentas LM e SS de forma integrada, no estudo de caso em uma empresa de acessórios automotivos na Índia. No artigo os autores apresentaram as aplicações das ferramentas LSS de forma clara e detalhada, e descreveram o sucesso obtido com as melhorias na aplicação LSS.

Spina (2007) apresentou um roteiro para aplicação LSS voltado ao setor de serviços e utilizou um sistema computacional de processos para auxiliar na agilidade dos dados e na preparação da aplicação do roteiro proposto. Um estudo de caso foi realizado em uma instituição financeira.

Rovet (2009) ilustrou as expectativas da integração LSS nas empresas, inclusive alguns erros recorrentes na sua implementação. No seu estudo de caso aplicou o LSS em uma empresa do setor de mineração. Os resultados obtidos no estudo de caso foram significativos quanto a melhorias na qualidade dos produtos e processos proporcionando um retorno financeiro.

Filho (2010) aplicou a metodologia LSS na redução da variabilidade do processo de envase em uma indústria de óleo vegetal. Através da metodologia foi 
possível evidenciar as principais causas da alta variabilidade no processo e propor as devidas soluções.

Silva et al. (2011) apresentou uma iniciativa de integrar LM e SS na busca de melhoria no desempenho operacional. 0 estudo de caso foi realizado em uma empresa fabricante de autopeças, os resultados indicaram que a iniciativa de conciliar as duas metodologias contribuiu para melhorar e tornar mais abrangente o efetivo processo de melhoria contínua na empresa estudada.

Grima (2012) estudou a aplicação das metodologias Seis Sigma e do Sistema Toyota de produção em indústria de pneus. Demonstrou as principais características de suas ferramentas, as particularidades a visão de gestão da qualidade aplicada a elas. No estudo de caso, o autor relatou das dificuldades encontradas na implementação das ferramentas, como também os motivos que foram determinantes para o sucesso de uma e fracasso de outra quanto à implementação.

\section{ROTEIRO PARA APLICAÇÃO DO LSS}

O roteiro proposto neste trabalho é inspirado em Spina (2007) que propôs um roteiro de aplicação LSS em uma empresa de serviços. O roteiro proposto por Spina (2007) estabeleceu um vínculo com método DMAIC para aplicação em um processo de serviço, com a utilização de um software de modelagem e simulação.

Neste trabalho foi elaborado um roteiro próprio mais amplo para possibilitar a aplicação do LSS em ambientes industriais. Segundo Assarlind et al.(2013) os benefícios do LSS podem ser alcançados através de uma abordagem padronizada que contemple o conceito de LSS de forma integrada.

O roteiro proposto é constituído de 5 etapas tendo como base o DMAIC e 11 passos detalhados, conforme ilustrado na Figura 4.

Figura 4 - Roteiro de aplicação Lean Seis Sigma

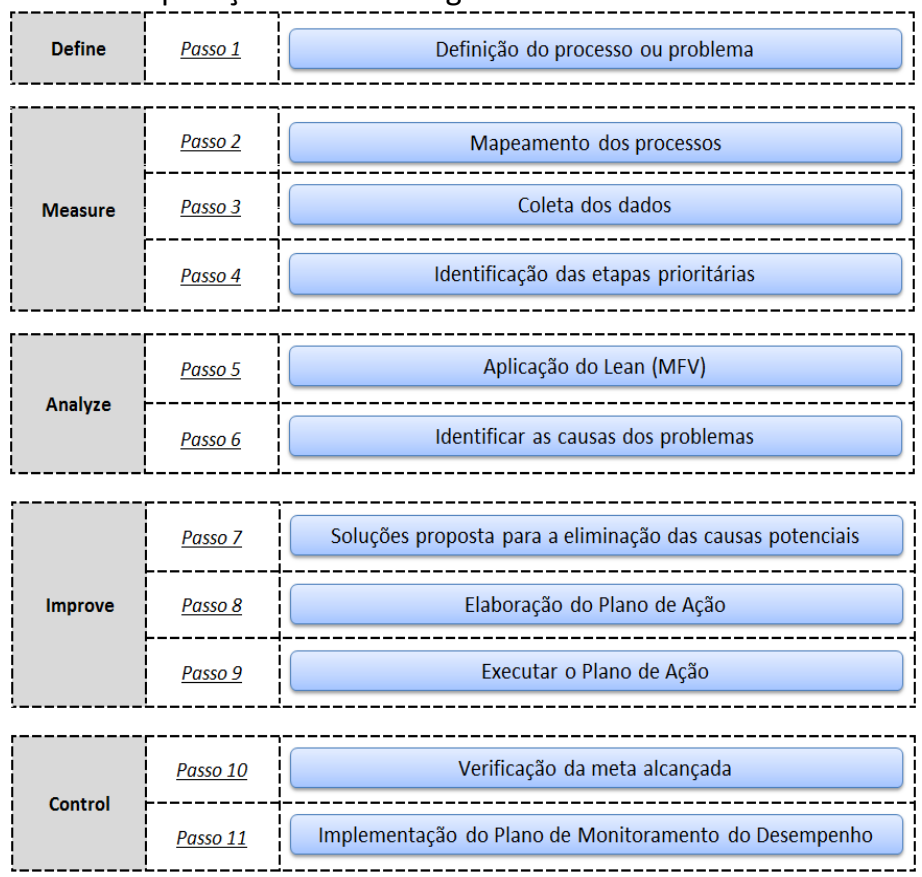

Fonte: Autoria Própria 
Etapa 1 (define): constituída apenas do Passo 1.

Passo 1: refere-se a definição do problema assim como a delimitação do(s) processo(s)/produtos envolvidos. Primeiramente é apresentada a problemática vivenciada pela organização, após o histórico do problema, assim comoa meta a ser alcançada. Em seguida, a definição do processo que pode ser melhorado, a fim de atender o propósito da empresa.

Etapa 2 (Measure): constituída dos Passos 2, 3 e 4.

Passo 2: Mapeamento do processo produtivo. Esse passo é essencial, pois permite conhecer o processo produtivo, analisar o detalhamento das atividades, entradas, saídas e os recursos utilizados.

Passo 3: Coleta de dados. Uma vez mapeado o processo produtivo necessitase coletar as informações qualitativas e quantitativas envolvidas no mesmo. Este passo permite gerar informações de suporte para o desdobramento do problema e a identificaçãode oportunidades de melhorias. Nesta etapa podem ser utilizadas as ferramentas propostas no centro da figura 3 , assim como as métricas utilizadas no Seis Sigma.

Passo 4: Identificação das etapas prioritárias do processo. A partir dos passos 2 e 3 será possível definir as principais etapas do processo produtivo envolvidas nas causas do problema em estudo no processo produtivo.

Etapa 3 (Analyze): constituída dos Passos 5 e 6.

Passo 5: Aplicado o Mapeamento de Fluxo de Valor no processo produtivo em estudo.

Passo 6: Identificação das causas do problema através da análise do MFV atual do processo produtivo em estudo.

Etapa 4 (Improve): constituída dos Passos 7, 8 e 9.

Passo 7: Análise e proposição de soluções para eliminação das causas potenciais do problema em estudo.

Passo 8: Elaboração de planos de ação voltados à eliminação das causas do problema que foram identificadas no Passo 7.

Passo 9: Realização dos planos de ação elaborados Passo 8.

Etapa 5 (Control): constituída dos Passos 10 e 11.

Passo 10: Verificação da meta alcançada após a realização dos planos de ação elaborados. Nesta etapa podem ser utilizadas as ferramentas propostas no centro da figura 3 assim como as métricas utilizadas no Seis Sigma,da mesma forma como foram utilizadas no Passo 2 afim de possibilitar a comparação dos resultados obtidos com os valores inciais.

Passo 11: Implementação de um plano de monitoramento de desempenho através de indicadores de variabilidade no processo que devem ser acompanhadas preiodicamente e apresentados em reuniões gerenciais 
Foi realizada neste trabalho, uma breve revisão teórica abrangendo conceitos do Lean Manufacturing, Seis Sigma e a integração das duas metodologias no Lean Seis Sigma. Além disso, buscou-se na literatura alguns casos de aplicação da integração LSS.

Neste trabalho foi proposto um roteiro original de 5 etapas e 11 passos para a aplicação do Lean Seis Sigma em ambientes industriais.

Com relação ao roteiro proposto a aplicação do Mapeamento do Processo no Passo 2 e posteriormente do Mapeamento do Fluxo de Valor no Passo 5 permite obter-se uma visão geral do processo, possibilitando elencar os principais etapas/desperdícios presentes no processo produtivo. A utilização integrada dos dois métodos de mapeamento torna possível e facilitada a identificação das perdas assim como a proposição de alternativas no fluxo de agregação de valor afim de solucionar o problema em estudo e alcançar melhorias significativas no mesmo.

Ressalta-se que a utilização das ferramentas propostas no centro da figura 3 assim como as métricas utilizadas no Seis Sigma podem ser utilizadas no Passo 2 e no Passo 10 afim de possibilitar a comparação dos resultados obtidos com os valores inciais. Através de análises comparativas será possível quantificar os resultados obtidos com relação a meta inicialmente estabelecida no Passo 1.

A escolha das ferramentas Lean Seis Sigma, torna-se um ponto essencial para aplicação do roteiro. A figura 3 ilustrou algumas das ferramentas disponíveis para uso na integração LSS. No entanto, necessita-se de conhecimentos práticos e teóricos de algumas das ferramentas disponíveis na literatura e perspicácia na escolha das mesmas.

As ferramentas utilizadas para definir e determinar os problemas podem ser gráficos sequenciais, diagrama de pareto, histogramas e mapeamento de processo. Na determinação das causas do problema as ferramentas podem ser o mapa do processo, brainstorming, diagrama de causa e efeito. Com relação às propostas de soluções para o problema, pode ser utilizado novamente o brainstorming, o plano de ação $5 \mathrm{~W} 1 \mathrm{H}$, Análise dos modos de falhas e seus efeitos, Failure mode and effects analysis (FMEA), entre outros.

Adicionalmente, para garantir que o alcance da meta seja mantido a longo prazo, pode ser elaborado um indicador de desempenho do processo através de alguma métrica sugerida no Seis Sigma. Sugere-se igualmente a possibilidade de utilização de uma carta de controle para o monitoramento do indicador elaborado. 


\title{
Lean six sigma implementation guide to improving industrial processes
}

\begin{abstract}
The concepts about Lean Manufacturing practices as well as the statistical tools of Six Sigma are widely diffused by the academic community. This paper aims to discuss the union of statistical concepts heavily exploited in Six Sigma allied to the waste elimination principles of Lean Manufacturing with the purpose of promoting the improvement of the productive process through the Lean Six Sigma approach. As a goal, this article seeks to present the concepts, methods, procedures of the stages and tools of quality proposed in Six Sigma, the tools proposed in Lean Manufacturing, as well as the integration of both in Lean Six Sigma. Some examples of the application of Lean Six Sigma will also be presented in case studies of the literature. After this, a roadmap will be proposed defining the steps to apply the integration of the Lean Six Sigma tools applied to obtain improvements in industrial processes.
\end{abstract}

KEYWORDS: Six Sigma. Lean Manufacturing. Lean six sigma. Industrial processes. 


\section{REFERÊNCIAS}

ASSARLIND, M.; GREMYR, I.; BACKMAN, K. Multi-faceted views on a Lean Six Sigma application. International Journal of Quality and Reliability Management, v. 30 , n. 4, p.387-402, 2013. crossref

EINSET, E., MARZANO, J. Six Sigma Demystified. Tooling \& Production, vol13, no.2, pp.43-47, abr. 2002.

FILHO, F. C. V.; ROSA, G. O.; MORALESD. Aplicação da metodologia LeanSix Sigma na redução da variabilidade do processo de envase em uma indústria de óleo vegetal. In: ENCONTRO NACIONAL DE ENGENHARIA DE PRODUÇÃO, 30., 2010, Bento Gonçalves. Anais eletrônicos... Bento Gonçalves: ENEGEP, 30. Disponível em: $<$ http://www.abepro.org.br/biblioteca/enegep2010_TN_STO_113_745_1663 1.pdf>. Acesso em 01 Set. 2015.

FONTE, M. O. A. O Lean Sigma aplicado uma indústria automobilística. Monografia de conclusão de Curso. Universidade Federal de Juiz de Fora, Minas Gerais, 2008. Disponível em:

<http:www.ufff.br/ep/files/2014/07/2008_1_Mariana.pdf Acesso em 21 set. 2015.

GEORGE, M. L. Lean Seis Sigma para serviços: Como utilizar a velocidade Lean e Qualidade Seis Sigma para Melhorar Serviços e Transações. Rio de Janeiro: Qualitymark, p.34, 2004.

GIANNINI, R. Aplicação de ferramentas do pensamento enxuto de redução de perdas em operação de serviços. Dissertação de Mestrado em Engenharia de Produção. Universidade de São Paulo, 2007. Disponível em: http://www.teses.usp.br/teses/disponiveis/3/3136/tde-10082007-174556/ptbr.php. Acesso em 19 maio 2015.

GREEF, A. C.; FREITAS, M. do C. D.; ROMAEL, F. B. Lean Office: Operação, Gerenciamento e Tecnologia. São Paulo: Atlas, 2012.

GRIMA. H.; CHAIN, R. Gestão da Qualidade: Aplicação do Seis Sigma e do Sistema Toyota de Produção em uma indústria de pneus. Monografia de Conclusão de Curso. Universidade Anhembi Morumbi. São Paulo, 2012. Disponível em: < http://engenharia.anhembi.br/tcc-11/prod-09.pdf>. Acesso em 26 ago. 2015.

KUMAR, M. et al. Implementing the Lean Sigma Framework in an Indian SME: A Case Study. PRODUCTION PLANNING \& CONTROL, v. 17, n. 4, p. 407-423, 2006. crossref 
KUNIYOSHI, D. S. Implementação da Metodologia Lean Seis Sigma em uma empresa do setor têxtil. Monografia de Conclusão de Curso. Escola Politécnica da Universidade de São Paulo. São Paulo, 2006. Disponível em:

http://pro.poli.usp.br/wp-content/uploads/2012/pubs/implementacao-dametodologia-lean-seis-sigma-em-uma-empresa-do-setor-textil.pdf. Acesso em 13 jun. 2015.

LÉXICO LEAN. Glossário ilustrado para participantes do pensamento lean. São Paulo: LeanInstitute Brasil, p. 39-57, 2003.

OHNO, T. O Sistema Toyota de Produção: Além da produção em larga escala. Porto Alegre: Bookman, p. 10-42, 1997.

PANDE, P.S., NEUMAN, R. P., CAVANAGH, R.R. Estratégia Seis Sigma: como a GE, a Motorola e outras grandes empresas estão aguçando seu desempenho. Rio de Janeiro: Qualitymark, p. 134-235, 2001.

PANNELL, A. Happy together: solid lean principles are at the heart of every successful six sigma program. Industrial Engineer, v.38, n. 3, p.46-49, Mar. 2006.

RAGO, S. F. Atualidades na Gestão de Manufatura. São Paulo: IMAM, p. 21-23, 2003.

REIS, D. A. F. dos. (2003). Seis Sigma: Um Estudo Aplicado ao Setor eletrônico. Dissertação de Mestrado, Universidade Federal do Rio Grande do Sul, Rio Grande do Sul, Brasil.Disponível em:

http://www.producao.ufrgs.br/arquivos/publicacoes/Delmar_Flemming.pdfAcess o em 14 jun. 2015.

ROTONDARO, R. G.Seis Sigma: Estratégia Gerencial para melhoria de processos, produtos e serviços. São Paulo: Atlas, p. 11-46, 2002.

ROVET, M. C. Programa Lean Seis Sigma: Uma visão plena de uma empresa do setor de mineração. In: ENCONTRO NACIONAL DE ENGENHARIA DE PRODUÇÃO, 33, 2009, Salvador. Anais eletrônico... Salvador: ENEGEP, 33. Disponível em:< http://www.abepro.org.br/biblioteca/enegep2013_TN_STP_178_015_22542.pdf >. Acesso em 15 set. 2015.

SHINGO, S. O sistema Toyota de produção do ponto de vista da engenharia de produção. 2. Ed. Porto Alegre: Artmed, p. 21-35, 1996. 
SILVA, I. B.; MIYAKE, D. I.; BATOCCHIO, A.; AGOSTINHO, O. L. Integrando a promoção das metodologias Lean Manufacturing e Six Sigma na busca de produtividade e qualidade numa empresa fabricante de autopeças. Revista Gestão \& Produção, Vol.18, N. 4, p. 687-704, 2011. crossref

SPINA, C. Aplicação de ferramentas Seis Sigma e Simulação Computacional ao aperfeiçoamento de Serviços: Roteiro de referências e estudo de caso. Dissertação de Mestrado profissionalizante em Administração de Empresas de São Paulo. Fundação Getúlio Vargas, São Paulo, 2007. Disponível em: < http://ti.srt.ifsp.edu.br/revistailuminart/index.php/iluminart/article/view/177>. Acesso em 18 jun. 2015.

WERKEMA, M. C. Criando a Cultura Seis Sigma. Rio de Janeiro: Qualitymark, p.1345, 2002.

WERKEMA, M. C. Perguntas e Respostas sobre o Seis Sigma: Série Seis Sigma,V. 6. Belo Horizonte: Editora Werkema, p. 9-31, 2008.

WERKEMA, M. C. Lean Seis Sigma: Introdução ás ferramentas do Lean Manufacturing. 2. Ed. Rio de Janeiro: Elsevier, p. 13-80, 2011. 